\title{
From maps to navigation: The role of cues in finding locations in a virtual environment
}

\author{
Adam T. Hutcheson • Douglas H. Wedell
}

Published online: 21 February 2012

(C) Psychonomic Society, Inc. 2012

\begin{abstract}
In two experiments, participants navigated through a large arena within a virtual environment (VE) to a location encoded in memory from a map. In both experiments, participants recalled locations by navigating through the VE, but in Experiment 2, they additionally recalled the locations on the original map. Two cues were located outside and above the walls of the arena at either north-south locations or east-west locations. The pattern of angular bias was used to infer how the cues affected the creation of spatial categories influencing memory for location in the two tasks. When participants navigated to remembered locations in the VE, two cue-based spatial categories were inferred, with cues serving to demarcate the boundaries of the categories. When participants remembered locations on the original map, two cue-based categories were again formed, but with cues serving as category prototypes. The pattern of results implies that cue-based spatial categorization schemes may be formulated differently at the memory retrieval stage depending on task constraints.
\end{abstract}

Keywords Categories · Spatial memory Cues · Retrieval · Category adjustment model

The ability to encode a location from a map into memory and then translate that location into a real-world coordinate system is an essential part of human navigation. There is abundant evidence that features of the environment are used to create categorical codes that bias memory for location when the retrieval environment matches the encoding environment (Huttenlocher, Hedges, \& Duncan, 1991; Spencer

\footnotetext{
A. T. Hutcheson $(\bowtie) \cdot$ D. H. Wedell

University of South Carolina,

Columbia, SC, USA

e-mail: hutchesa@mailbox.sc.edu
}

\& Hund, 2002; Wedell, Fitting, \& Allen, 2007). However, recent research has indicated that one key determinant of spatial memory performance is whether one must shift points of view between encoding and retrieval (Friedman, Waller, Thrash, Greenauer, \& Hodgson, 2011; Greenauer \& Waller, 2008, 2010; Shelton \& McNamara, 2004; Waller, 2006; Waller, Friedman, Hodgson, \& Greenauer, 2009). The research we present here explored how shifting perspective affects biases in spatial memory performance. In particular, we investigated how locations from a map that were encoded in memory would be recalled when participants navigated through a computer-simulated virtual environment (VE) corresponding to the map. Our primary experimental manipulation was the location of cues within the environment. We examined whether cues are used to encode memory for location when one navigates through an environment from an egocentric perspective in the same way as when one views a small-scale representation (such as a map) from an allocentric point of view (Fitting, Wedell, \& Allen, 2007a, b, 2008a, b).

Extant evidence supports the idea that people parse spatial layouts into categories and adjust their estimates of location toward the central tendency of the corresponding spatial category, as depicted in Fig. 1 (Fitting, Wedell, \& Allen, 2007a, b, 2008a, b; Huttenlocher, Hedges, Corrigan, \& Crawford, 2004; Huttenlocher et al., 1991; Spencer \& Hund, 2002; Verbeek \& Spetch, 2008; Wedell et al., 2007). Results from this research are generally consistent with the category adjustment model of spatial memory proposed by Huttenlocher and colleagues (Huttenlocher et al., 1991), according to which spatial memory is encoded at two levels: (1) fine-grain memory based on distance and direction of a target location from a reference point and (2) categorical memory based on a gross partitioning of the environment into coherent components. Fine-grain memory is assumed to 

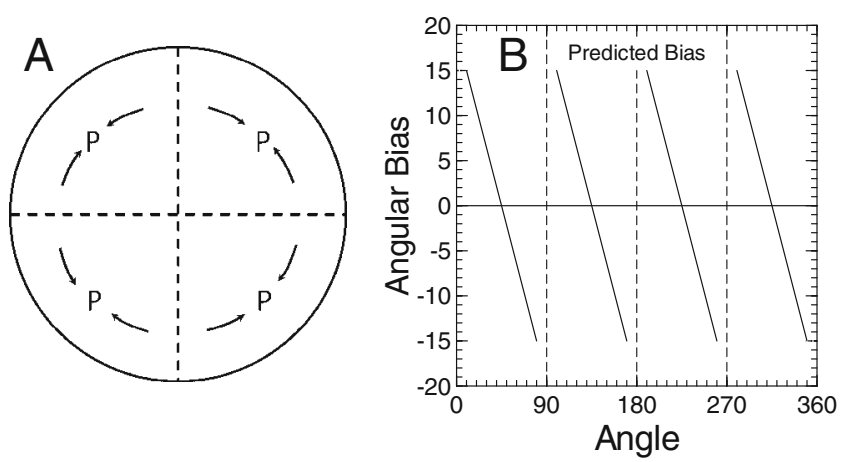

Fig. 1 Depiction of angular bias effects for dot in circle task. a Four quadrant prototypes $(\mathrm{P})$ toward which estimates are biased. b Resulting predicted angular bias pattern from the category adjustment model

be more fragile than categorical memory. Accordingly, research has demonstrated that forgetting induced by delays or interference tasks results in estimates that are more dependent on categorical encoding and, hence, reflect greater bias toward category prototypes (Engebretson \& Huttenlocher, 1996; Fitting, Wedell, \& Allen, 2007a, b; Hund \& Plumert, 2002; Huttenlocher et al., 1991). Figure 1 shows typical results for the basic dot-in-circle task, in which participants briefly view a location in a homogeneous spatial layout and then, a few seconds later, reproduce this location. Participants appear to divide the circle into four quadrants, and remembered locations are shifted toward the central prototypes for each quadrant, as shown in panel A, with the resultant pattern of angular bias shown in panel B.

Most of the studies investigating the category adjustment model have examined spatial memory estimation when participants were stationary within their environment. As a preliminary examination of categorical effects upon navigation, Fitting, Allen, and Wedell (2007a, b) demonstrated results consistent with predictions of the category adjustment model in a human analogue to the Morris water maze, in which participants placed a marker on the floor of a 3-mdiameter arena at the remembered location. Their results indicated cue-based prototypes and demonstrated that the predicted categorical bias can be found in a traversable space.

Fitting and colleagues have also investigated how the nature of cues may or may not affect how spatial categories are defined. Using the basic dot-in-circle task, Fitting and colleagues found that cues surrounding the task field were largely ignored as long as the task field remained stationary and memory was not overly taxed. When participants had to remember multiple targets or if the task field was rotated on a majority of trials, categories were centered on salient cues, rather than reflecting the four-quadrant division (Fitting, Wedell, \& Allen, 2007a, b, 2008a, b).

One underexplored area of research is how categorical encoding may affect navigation. Several issues arise in this regard. First, frame of reference may become an important factor, since previous research has demonstrated that people may form multiple representations of an environment depending on task demands and encoding conditions (Friedman et al., 2011; Greenauer \& Waller, 2008, 2010; Mou \& McNamara, 2002; Mou, McNamara, Rump, \& Xiao, 2006; Shelton \& McNamara, 2004; Waller, 2006; Waller et al., 2009). Specifically, people are not limited to the viewpoint representation of an environment from which it was learned. When the task requires the use of a viewpoint that was not previously experienced during encoding, people may construct a representation based on the available information that allows them to make judgments about the environment. Much of the work on spatial category biases utilizes an allocentric frame of reference, as participants view relationships among objects, rather than relative to their own orientation. Because navigation typically involves moving through a large-scale environment, it primarily utilizes a viewer-based or egocentric frame of reference. ${ }^{1}$ Will the same biases as those observed for allocentric orientation also be found for egocentric orientation? While the work of Haun, Allen, and Wedell (2005) supports this generalization, that study did not involve navigation but, rather, had participants point to locations relative to their own fixed location.

Second, related to this issue is the effect of scale of environment. Much of the research on spatial category biases has been conducted on a small two-dimensional display, such as a computer screen. Will similar category biases be observed when the relevant task field is not viewable in a glance and must be navigated? While the Fitting, Allen, and Wedell (2007a, b) human analog to the Morris water maze study supports this generalization, the task space in that experiment was relatively small and required only minimal movement. The scale of the task space and the potential for disorientation through movement have been found to be significant factors in spatial performance in past research examining developmental differences (Learmonth, Nadel, \& Newcombe, 2002; Learmonth, Newcombe, \& Huttenlocher, 2001; Learmonth, Newcombe, Sheridan, \& Jones, 2008).

Third, related to both of these issues is whether the way information about location is acquired or encoded will affect how it is expressed at retrieval. For example, one could encode a location via a map and then navigate to it, or one could encode that location through the experience of navigating through the environment. Thorndyke and Hayes-

\footnotetext{
${ }^{1}$ It is possible for an aerial perspective to be an egocentric perspective. For example, if a person is looking at a city from the roof of a skyscraper, he or she would have an aerial egocentric view. Our study does not focus on this type of situation. We assume that the navigational condition taps into an egocentric perspective and the map condition an allocentric perspective.
} 
Roth (1982) have shown that maps allow people to easily use an overview of the environment (survey knowledge) to find locations and remember distances. Direct experience requires a person to first use memory for landmarks in a sequence (route knowledge) before eventually gaining survey knowledge (Ishikawa \& Montello, 2006; Siegel \& White, 1975). An advantage of learning from a map is that it provides an overview of the environment that aids relational encoding; however, an advantage of learning through navigation is that there is no need to switch from an allocentric to an egocentric frame of reference. Maps typically also provide a stable orientation to the environment, unlike navigating from an egocentric view, which is very dynamic (Shelton \& Pippitt, 2007). One general conclusion from this research is that people benefit from maintaining the same orientation and task goals (route or survey goal) during retrieval that were present during encoding (Taylor, Naylor, \& Chechile, 1999). If one must switch between two frames of reference, there is a price to pay in terms of speed and accuracy (Shelton \& McNamara, 2004). This conclusion is consistent with research demonstrating that memory for object arrays is best when tested using similar orientations at both encoding and retrieval (Diwadkar \& McNamara, 1997; Friedman \& Waller, 2008; Friedman et al., 2011; Waller et al., 2009).

Finally, an important issue in considering how categorical bias is incorporated in navigation is the role of cues or landmarks. Clearly, landmarks play several critical roles in navigation, such as helping to determine an initial heading, helping to correct headings, and determining proximity to the target, among other functions (Denis, Michon, \& Tom, 2007). The literature on memory for spatial locations also suggests that landmarks may be critical to categorical encoding and, hence, may bias navigation. In support of this assertion, Fitting, Wedell, and Allen (2009) investigated the role of cues in navigating in a two-dimensional maplike environment that combined features of the dot-in-circle task with features of the Morris water maze. In that study, human participants used a mouse to control a computer simulation of a rat swimming in the water from an aerial perspective (allocentric orientation). They found that in addition to large effects of starting position and task field orientation, cue locations significantly affected initial heading, efficiency of navigation, and end location bias in ways consistent with a version of the category adjustment model in which cues serve as category prototypes, biasing initial heading and search locations toward them. Although the results of Fitting et al. (2009) support the generalization of spatial biases from the dot-in-circle task to navigation tasks, features of their experimental paradigm raise questions of how general these effects may be. First, because they used a maplike environment, it is unclear whether results would generalize to a traversable environment viewed from an egocentric perspective. Furthermore, while orientation to the task field changed via rotation, the allocentric framework was maintained throughout the study. Thus, it is unclear how encoding a location from an allocentric framework, such as seeing the location on a map, would translate to navigating to the location using an egocentric framework.

We explored these issues in two experiments. Participants encoded the target locations from an allocentric perspective by viewing the location on a map. In Experiment 1, participants first learned the location from a map presented on a computer and then played a video game on another computer for a minute before navigating to the corresponding location in a VE presented on a large central screen. Because transforming memory encoding from an allocentric map representation to an egocentric orientation used in navigation is memory taxing, we hypothesized that the map-based encoding used in these experiments would produce large cue-based angular bias effects. In Experiment 2, participants responded both on maps and in the VE. We expected that the VE data would replicate the Experiment 1 results, showing cues that serve as category boundaries with prototypes in the center of the categories, but that the map data would show cue-based categories with the prototypes centered near the cues, consistent with prior research using a similar task environment (Fitting, Wedell, \& Allen, 2007a, b). In both experiments, the locations of two cues were varied in order to assess the cue dependence of the expected categorical biases.

\section{Experiment 1}

\section{Method}

Participants Forty-nine (35 female and 14 male) undergraduate students from the University of South Carolina participated in this experiment. They were recruited from the psychology department participant pool and received class credits for their voluntary participation. None of the participants reported having extensive experience playing firstperson shooter video games. ${ }^{2}$

Design and materials The design consisted of a 2 (cues) $\times$ 8 (target angle) $\times 2$ (target radius) factorial design with the first variable manipulated between subjects and the other variables manipulated within subjects. Computers were used to present all materials and track navigational coordinates. The VE was made using First Person Shooter Creator (The Game Creators (C1999-2011). This program allows one to

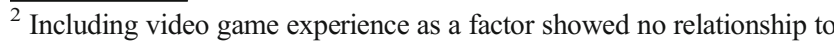
performance for any main effects or interactions reported below, so it was not included in our reporting of results for either Experiment 1 or 2.
} 
quickly build VEs and easily get coordinates of location within the VE. The VE used for estimates of location was projected on a wall approximately $0.86 \mathrm{~m}$ in front of the participant, using a multimedia projector. The setup used in Experiment 1 is depicted in Fig. 2. The projected image was approximately $1.83 \times 1.22 \mathrm{~m}$ in area and $0.51 \mathrm{~m}$ above the desk at which the participant was seated. Distal cues appeared in the "sky" at either the north and south positions or the east and west positions of the arena. The dependent variable was the estimated location of each target location, recorded in Cartesian coordinates and converted to polar coordinates. Participants experienced one of four randomly generated presentation orders to control for order effects.

As is shown in Fig. 2, the computer to the participant's right was used to present the map location of the target, and the computer to the participant's left was used to present a video game that was played between map encoding of location and VE navigation to the location. The game was a simple public domain video game called "Attack of the Buggles," in which participants maneuvered an icon across the screen to capture points while avoiding running into icons that would terminate the game. This type of spatial navigation game was used in order to interfere with spatial memory for the target location and, thus, create greater error and bias that constitute the primary basis for inferring spatial categories. The mouse on the participant's left was used to control the video game, and the mouse in front of the participant was used to navigate through the projected VE. Maps consisted of a $380 \times 380$ pixel array and were

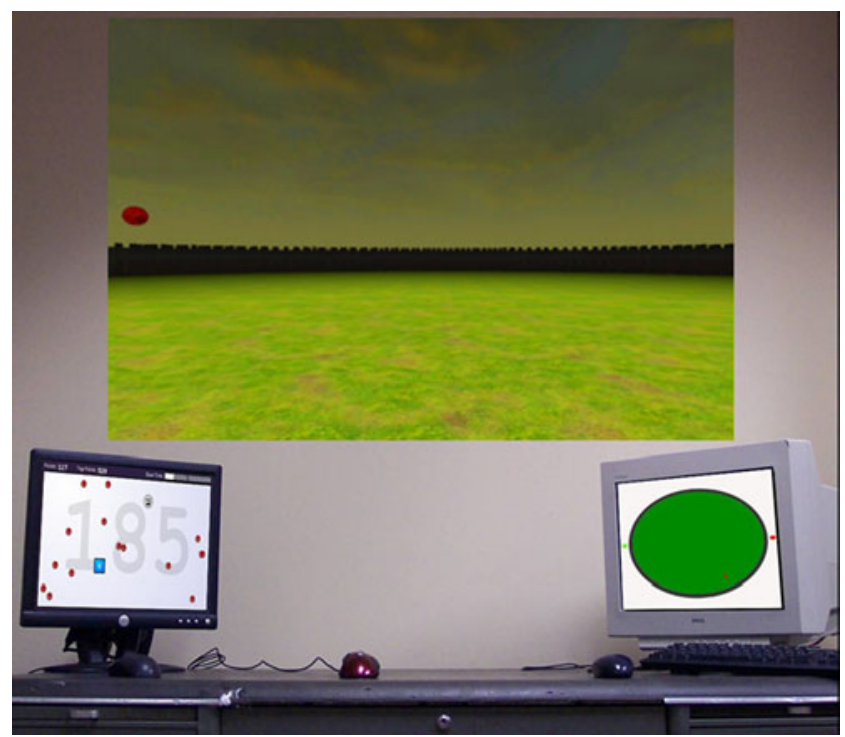

Fig. 2 Experimental setup with map locations presented on the right computer screen, the video game presented on the left computer screen, and the virtual environment (VE) projected on the wall in front of the participant. Participants first studied the location of the dot on the map and then played the video game for $1 \mathrm{~min}$ before navigating to the location in the VE presented in a slide show using Microsoft PowerPoint. Sixteen target locations were established around the interior of the arena at angular locations of $30^{\circ}, 75^{\circ}, 120^{\circ}$, $165^{\circ}, 210^{\circ}, 255^{\circ}, 300^{\circ}$, and $345^{\circ}$. The long-radius targets had a radial distance of 1,100 units, or $66 \mathrm{ft}$, from the center of the arena. The short-radius targets had a radial distance of 600 units, or $36 \mathrm{ft}$, from the center of the arena. Two versions of the slide shows were used to create the between-subjects factor of cue location. Half of the participants saw a red circle at the top of the screen and a green square at the bottom of the screen (northsouth $[\mathrm{NS}])$. The other half saw the red circle on the right side of the screen and the green square on the left (eastwest $[E W])$. These external cues corresponded to NS and EW cue locations in two different versions of the VE that were otherwise identical.

Procedure Participants were tested one at a time in a dimly lit room, with an experimenter sitting beside them and guiding them through the procedures. Participants were given time to practice using the mouse to navigate the $\mathrm{VE}$ and to understand how the video game worked. They completed a practice trial on which they saw a sample map and then played the video game for $1 \mathrm{~min}$ before attempting to find the location of the target in the VE projected on the wall. If they understood the procedure, they were allowed to begin the first trial. On each trial, they studied the map for $30 \mathrm{~s}$ until it disappeared from the computer screen. An on-screen instruction told them to turn and play the video game. They did so for $1 \mathrm{~min}$ until a chime sounded, and then they used the mouse to navigate from the center of the VE to where they believed the target location was. Navigation in the VE for all conditions started with the viewpoint facing north. After completing a trial, the participant studied the next map while the experimenter recorded the observed location by opening the program's debugging screen in order to access the coordinates of the current position. Then they reset the starting position at the center of the arena.

\section{Results}

Recalled locations that are very deviant from the actual location may arise from gross mistakes of misremembering, such as recalling a previous target rather than the current one or remembering the location as close to the green cue when it was close to the red cue. These large discrepancies in recalled position obscure biases occurring for participants who recall the general location well. Thus, we first screened the data by the criterion that the remembered location had to be within 750 units of the actual location. This distance corresponded to half the radius of the arena. Participants who had more than four such errors were dropped, resulting in the elimination of 3 participants, so that 46 were retained of the original 49. For these participants, individual data 
points were removed if they did not meet the criterion above. This resulted in $4.35 \%$ of the data being eliminated. For repeated measures ANOVAs, these eliminated data points were replaced by the mean value for the corresponding condition. Because of the moderately large number of replaced data, the significance criterion was set at .01 for significance tests in this experiment. Prior to running the reported ANOVAs, we ran ANOVAs that included sex as a betweensubjects factor (33 female and 13 male). Because none of the interactions with the sex variable were significant (and due to the unbalanced sample size), we dropped sex from the analyses reported below. The dependent measures were calculated as follows: (1) Angular bias equals the angle from the center to the observed location minus the angle from the center to the actual location, (2) radial bias equals the pixel distance from the center to the observed location minus the distance from the center to the actual location, and (3) absolute error equals the pixel distance from the observed location to the actual location.

Angular bias Angular bias scores are shown in Fig. 3 as a function of cue condition, along with the fit of the category adjustment model. Unlike the standard four-quadrant-based prototype pattern shown in Fig. 1, the pattern in Fig. 3 is consistent with an underlying two-category structure that shifts markedly across cue conditions. For the EW condition shown in the top panel, prototypes appear to be located close to the north $\left(90^{\circ}\right)$ and south $\left(270^{\circ}\right)$ positions (since prototype locations correspond to the angle at which the downward sloping lines cross the zero bias value). This pattern is consistent with EW cues forming a border that partitioned the space into north and south hemispheres, with the prototype centered in each hemisphere. For the NS condition shown in the bottom panel, prototypes tend to be located close to the east $\left(0^{\circ}\right)$ and west $\left(180^{\circ}\right)$ positions. Again, this pattern is consistent with NS cues forming a border that partitioned the space into east and west hemispheres, with the prototype centered in each hemisphere.

A 2 (cues) $\times 2$ (radius) $\times 8$ (angle) repeated measures ANOVA was conducted on angular bias scores. The main effect of angle was significant, $F(7,301)=5.86, p<.001$. However, there was also a very large cues $\times$ angle interaction, $F(7,301)=7.64, p<.001$, with significant trends for the 2nd-, 3rd-, and 5th-degree polynomial contrasts (each $p<.01$ ). This interaction provides clear support for a different categorical structure imposed on the target locations across the different cue conditions.

To describe the relationship and determine prototypes, the basic category adjustment model was fit to the angular bias data as follows:

$\operatorname{Bias}=\lambda \mu+(1-\lambda) \rho_{\mathrm{j}}-\mu$
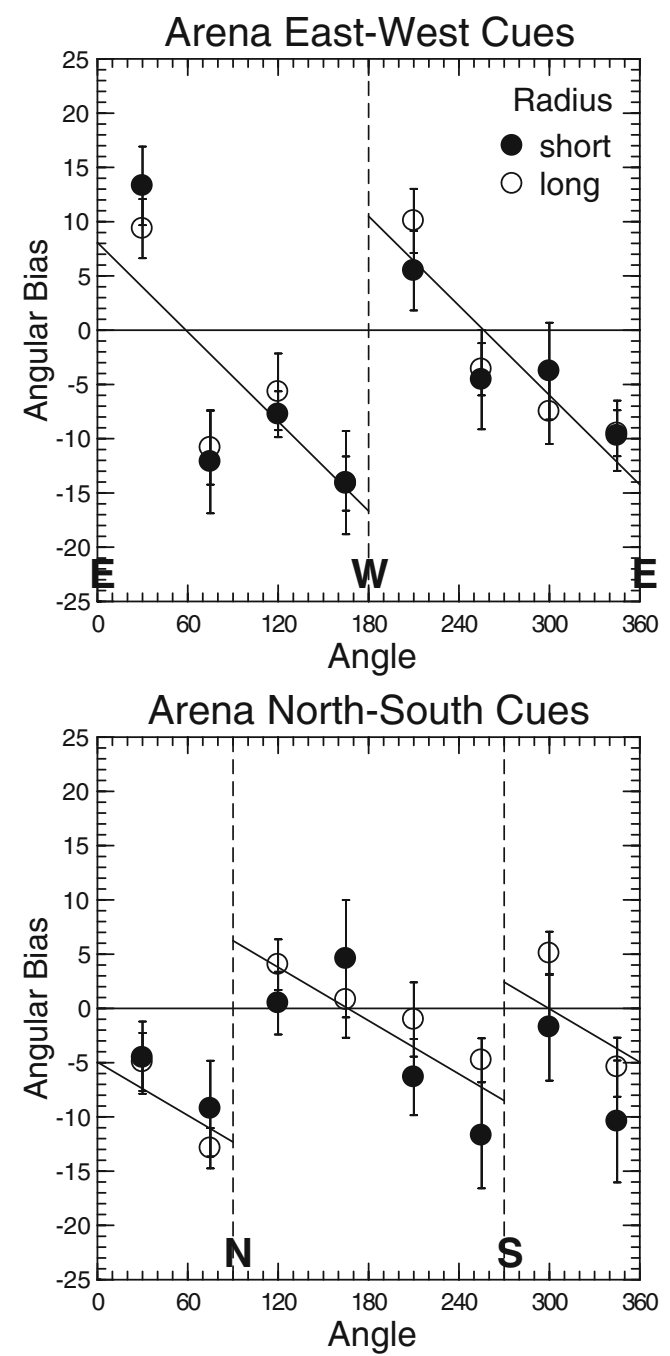

Fig. 3 Mean angular bias (with standard error bars) as a function of target angle and cue location in Experiment 1. Letters (E, W, N, S) reflect locations of east, west, north, and south cues, respectively. Results are consistent with two prototypes falling between the presented cues. Prediction lines are from the fit of the category adjustment model

where $\mu$ is the actual angle of the target (as represented by the fine-grain memory mean), $\lambda$ is the relative weighting of fine grain memory, and $\rho_{\mathrm{j}}$ is the prototype value for the relevant quadrant. For the EW condition, one prototype was recruited for quadrants 1 and 2, and another prototype was recruited for quadrants 3 and 4 . For the NS condition, one prototype was recruited for quadrants 4 and 1 , and another prototype was recruited for quadrants 2 and 3. Separate model fits were obtained for EW and NS cue conditions, each with three parameters free to vary $\left(\lambda, \rho_{1}\right.$, and $\left.\rho_{2}\right)$. Note that $\lambda$ determines the slope of the bias functions, and the prototype values determine $x$-intercepts for each function. This model fit reasonably well, using $R^{2}$ as an index of fit. For the EW cues condition, $R^{2}=.674$, with fine-grain memory weight $\lambda=0.862, \rho_{1}=58.75$ for quadrants 1 and 
2 , and $\rho_{2}=256.48$ for quadrants 3 and 4 . For the NS cues condition, $R^{2}=.658$, with fine-grain memory weight $\lambda=$ $0.918, \rho_{1}=166.19$ for quadrants 3 and 4 , and $\rho_{2}=299.50$ for quadrants 4 and 1 .

Radial bias A repeated measures ANOVA of the same form as that described above was conducted on radial bias. The only effect to achieve statistical significance at $p<.01$ was the main effect of radius, $F(1,44)=20.08$, $p<.001, \eta^{2}=.313$. While both short- and long-radius targets were underestimated, underestimation was significantly greater for the long-radius targets $(M=-180.64)$ than for the short-radius targets $(M=-107.36)$, which were closer to the central starting point for navigation. These results are consistent with underestimation of distances in VE environments (Foreman, Sandamas, \& Newson, 2004; Hutcheson \& Wedell, 2009) and appear to have a similar form in that the further the actual distance, the greater the underestimation.

Absolute error A repeated measures ANOVA of the same form as that described above was conducted on absolute error. The only significant effect at $p<.01$ was the main effect of radius, $F(1,44)=28.92$. The average absolute error was greater for long-radius targets $(M=313.61)$ than for short-radius targets $(M=244.07)$. This effect is likely due to the greater radial bias for longer targets.

\section{Discussion}

The results of Experiment 1 were consistent with the idea that the participants used the cues to create an axis of orientation to the arena rather than center category prototypes on the cue locations. Although this axis could be used to create a four-quadrant categorical partitioning of the space (which would have resulted in the same pattern of bias across cue conditions), participants appeared to use hemispheric partitioning so that only two prototypes were created in each cue condition. The two prototypes in each case were approximately centered within the hemisphere and, hence, were rotated approximately $90^{\circ}$ across cue conditions. This distinct pattern of bias is presented in Fig. 3 and is consistent with an application of the category adjustment model with a two-prototype configuration.

The cue dependence of spatial categories was not surprising in this case, because prior research implied that when the task field must be transformed, external cues are likely to be used to generate cue-based categories (Fitting, Wedell, \& Allen, 2007a, b, 2008b, 2009). In those studies, the transformation was the rotation of the task field relative to the viewer. Experiment 1 extended these findings to include transformation from an allocentic (map) representation to an egocentric (VE) representation. What is surprising, however, was that the cue-based categories were determined by using the cues as axial boundaries, with prototypes centered in between cues, rather than using the cues as prototypes themselves, as was found in the previously cited research. The key differences between the two streams of research is that in the earlier studies, a small-scale environment with allocentric orientation was maintained, whereas in the present experiment, the small-scale allocentric orientation was translated to a large-scale egocentric orientation in order to navigate through the VE. These results, when combined with past research, imply that cues are used differently when estimating memory from within allocentric and egocentric frames. Experiment 2 was designed to determine whether the categorical structure found in navigating within the VE would extend to locating positions back on the allocentric map or whether these distinctly different bias patterns could be found for the same participants within the same experiment.

Experiment 1 also demonstrated effects on radial bias consistent with the idea that distances are underestimated when navigating in a VE (Foreman et al., 2004; Hutcheson \& Wedell, 2009; Witmer \& Sadowski, 1998). In Experiment 1 , the starting point was always the center of the arena, so underestimation would result in negative bias. Both shortand long-radius targets were underestimated. Furthermore, the underestimation was greater for the more distant longradius targets. This pattern of increased underestimation with distance traveled is consistent with effects reported in the previously cited research.

\section{Experiment 2}

The specific nature of the cue-based categories found in Experiment 1 was not anticipated by the previous research. In that research, spatial categories were centered on cue locations, whereas, in Experiment 1, cues served to define the boundaries of the categories. The key difference between these lines of research would appear to be whether the orientation to the task space was allocentric or egocentric. In the egocentric orientation, distal cues may serve to orient the viewer, and hence, it may be natural to align category boundaries with such cues. In the allocentric orientation, the cues may represent distal objects, but they may be viewed as proximal to targets. As such, targets may be lined up with cues, and hence, cues serve as category prototypes.

Experiment 2 was designed to directly test whether cues are used differently in finding locations when the orientation to the environment is allocentric rather than egocentric. Specifically, it may be possible to show different representations of spatial location when retrieval uses different perspectives even though judgments are made within seconds 
of one another and the encoding condition is the same. Thus, Experiment 2 explored the degree to which the task space at retrieval affects how spatial categories are defined and used. The method was the same as that used in Experiment 1, except for one key difference. In addition to navigating the VE to indicate the remembered location, the participants also indicated the remembered location on the map. The order of the two tasks was counterbalanced across participants, so that half navigated the VE before recalling the location on the map and the other half did these tasks in the reverse order. We predicted that performance for the VE location task would replicate that of Experiment 1, in which categories are cue dependent but the cues are used to define category boundaries. In contrast, we anticipated that cuebased category prototypes would be observed in map recall.

\section{Method}

The method of Experiment 2 differed from that of Experiment 1 in only a few respects. Seventy-nine (54 female and 25 male) previously untested undergraduates were recruited from the same pool as before and received class credits for their voluntary participation. Once again, the large majority of participants had little or no experience with first-person shooter games. The design consisted of a 2 (response order) $\times 2$ (cues) $\times 8$ (target angle) $\times 2$ (target radius) factorial design, with response order and cues manipulated between subjects and the other variables manipulated within subjects. The VE used in Experiment 1 was used for this experiment without any changes. We also used the same video game between trials. The only change in the materials was in our choice of presentation software for the maps. We used E-Prime software (Psychology Software Tools, Sharpsburg, PA), which enabled us to have participants use the mouse pointer to indicate locations on blank maps in addition to navigating the VE. The procedure remained the same as that in Experiment 1, with one notable exception. Since we were interested in obtaining responses on maps as well as in VEs, half of our participants recalled the target locations on blank versions of the maps (containing either NS cues or EW cues) and then gave an estimate of location in the VEs, and the other half responded in the VEs prior to recalling the location on the map. For the map location task, participants were required to use a computer mouse to click within the circular field of the map, and E-Prime recorded coordinates for their estimates. These estimates were used in the analysis of angular and radial bias just as was done for estimates in the VE.

\section{Results}

Parallel analyses were conducted for VE recall and map recall. For VE data, a data point was eliminated if the remembered location was more than 750 units away from the actual location, the same criterion as in Experiment 1. A parallel criterion was used for the map data; however, because each pixel on the map was equal to 10 units in the VE, the cutoff was set at 75 rather than 750 . For the full data set, participants with more than four missing data points in either the VE or the map task were dropped from the analysis. This resulted in dropping 9 participants, leaving a sample size of 70 . For repeated measures ANOVAs, these eliminated data points were replaced by the mean value for the corresponding condition. The percentage of points replaced with corresponding conditional means was $5.0 \%$ for the VE data and $2.4 \%$ for the map data. Because of the moderately large number of replaced data, the significance criterion was set at .01 for significance tests in this experiment. Once again, prior to running the reported ANOVAs, we ran ANOVAs that included sex as a between-subjects factor ( 45 female and 25 male). Although there were some interactions with the sex variable, none of these were with key factors on our design. Given the highly unbalanced sample sizes for males and females and the lack of interaction effects, we dropped sex from the analyses reported below.

Angular bias for VE localization Figure 4 illustrates the pattern of angular bias found for VE localization as a function of cue condition and task order. The pattern of angular bias when the VE location was estimated first (left panels) is very similar to that obtained in Experiment 1, once again reflecting two category prototypes that shift markedly across cue conditions. As in Experiment 1, the cues appear to serve as boundary markers, and the prototype locations are near the centers of the spaces partitioned by the cues. The pattern of angular bias effects when map locations were estimated just prior to the VE task is somewhat different. The EW cue condition shows a similar effect for both task orders, with two spatial categories bounded at the cue locations $\left(0^{\circ}\right.$ and $\left.180^{\circ}\right)$. The NS cue condition, on the other hand, shows different patterns for the two task orders. When VE is estimated first, a two-category model seems appropriate, with boundaries defined by the cues. However, when map locations were estimated first, the pattern for VE estimation is closest to a four-quadrant pattern.

A 2 (order) $\times 2$ (cues) $\times 2$ (radius) $\times 8$ (angle) repeated measures ANOVA was conducted on angular bias scores for the VE localization task. The main effect of angle was significant, $F(7,462)=5.59, p<.001$. There was also a very large cues $\times$ angle interaction, $F(7,462)=5.85, p<$ .001 , indicating that cues affected the nature of the angular bias. These effects of cues on angular bias were further moderated by task order, as reflected in the significant order $\times$ cues $\times$ angle interaction, $F(7,462)=2.83, p<.01$. Thus, the nature of the two-way interaction of cues and 
Fig. 4 Mean angular bias (with standard error bars) in the virtual environment (VE) localization task as a function of target angle, cue location, and task order in Experiment 2. Letters (E, W, N, S) reflect locations of east, west, north, and south cues, respectively. Results are generally consistent with two prototypes falling between the presented cues, except for the map-first northsouth cues condition. Prediction lines are from the fit of the category adjustment model
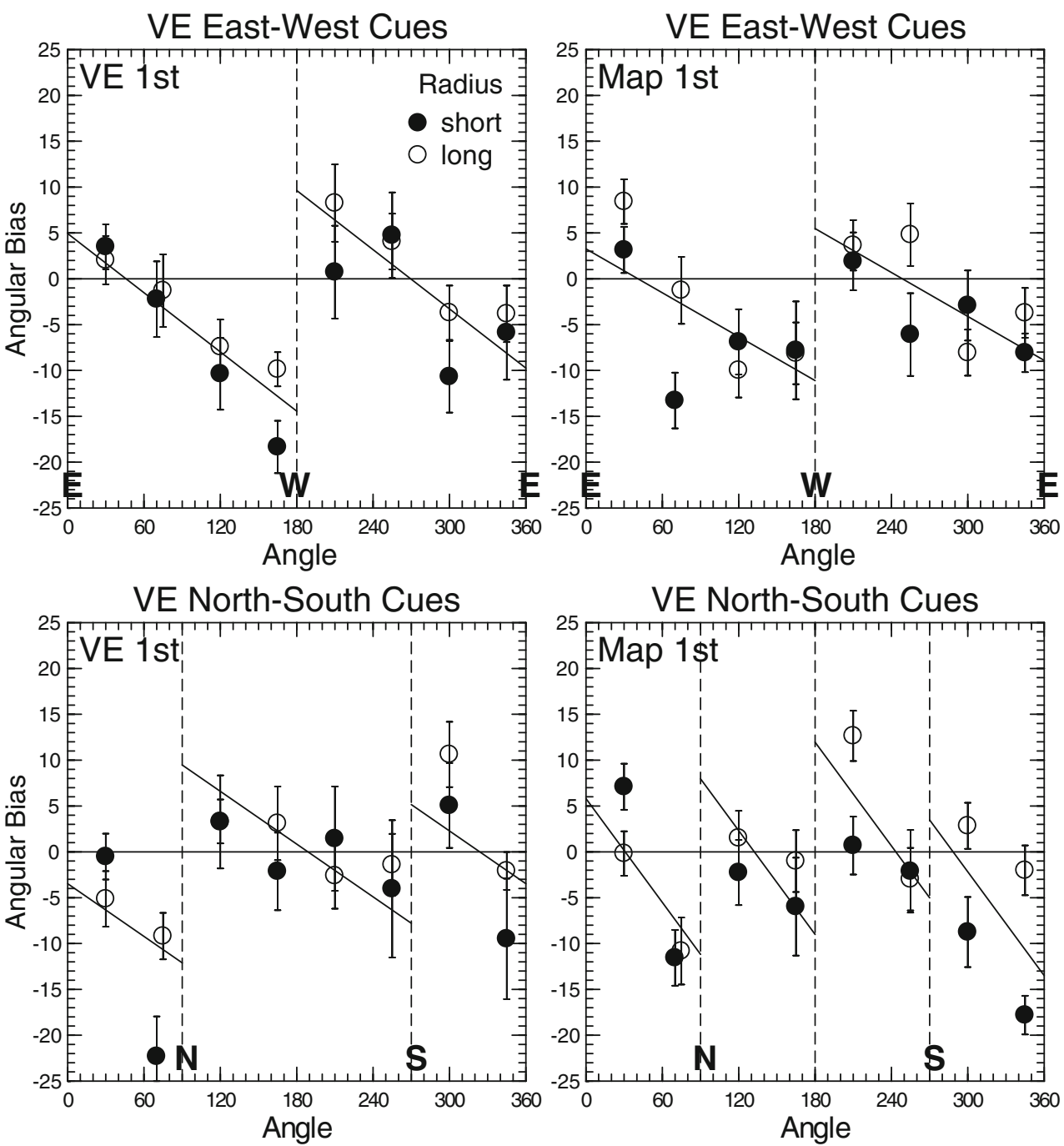

angle depended on whether VE estimation occurred first or followed map estimation. Two other effects reached statistical significance. The main effect of radius, $F(1,66)=$ $21.25, p<.001$, reflected more positive overall bias scores for the long radius relative to the short radius. Thus, there was a tendency for the long-radius targets to be recalled somewhat counterclockwise from the recalled locations of corresponding short-radius targets. The only other significant effect was a radius $\times$ angle interaction, $F(7,462)=3.17$, $p<.01$, indicating that the effect of radius on angular bias was not uniform across angles. Of the eight target angles, only target angle $1\left(30^{\circ}\right)$ did not show a more positive bias for long- than for short-radius targets.

To better describe the order $\times$ cues $\times$ angle interaction demonstrated above, we fit the basic category adjustment model of Eq. 1 to the angular bias data for each cue $\times$ order condition in three ways: (1) using the four-quadrant model (consisting of five free parameters: $\lambda, \rho_{1}, \rho_{2}, \rho_{3}$, and $\rho_{4}$ ), (2) using the two-quadrant model partitioned at $0^{\circ}$ and $180^{\circ}$ (consisting of three free parameters: $\lambda, \rho_{1}$, and $\rho_{2}$ ), and (3) using the two-quadrant model partitioned at $90^{\circ}$ and $270^{\circ}$ (also consisting of three free parameters: $\lambda, \rho_{1}$, and $\rho_{2}$ ). For all three models, the prototypes were constrained to fall within the designated category partitions so that the bias function must cross the $x$-intercept within the partition. The regression functions for the model that fit best in each condition are shown in Fig. 4. For the EW-VE-first condition, the two-category model with boundaries at $0^{\circ}$ and $180^{\circ}$ fit best, $R^{2}=.757, \lambda=0.892, \rho_{1}=45.77$, and $\rho_{2}=269.44$. This same model fit best for the EW-map-first condition, $R^{2}$ $=.482, \lambda=0.920, \rho_{1}=40.86$, and $\rho_{2}=248.54$. For the NSVE-first condition, the two-category model with boundaries at $90^{\circ}$ and $270^{\circ}$ fit best, $R^{2}=.522, \lambda=0.904, \rho_{1}=188.70$, and $\rho_{2}=323.78$. However, for the NS-map-first condition, the four-quadrant model fit best, $R^{2}=.529, \lambda=0.811, \rho_{1}=$ $30.76, \rho_{2}=132.24, \rho_{3}=243.42$, and $\rho_{4}=288.38$.

Angular bias for map localization Figure 5 illustrates the pattern of angular bias found for map localization as a function of cue condition combining across task order. The 

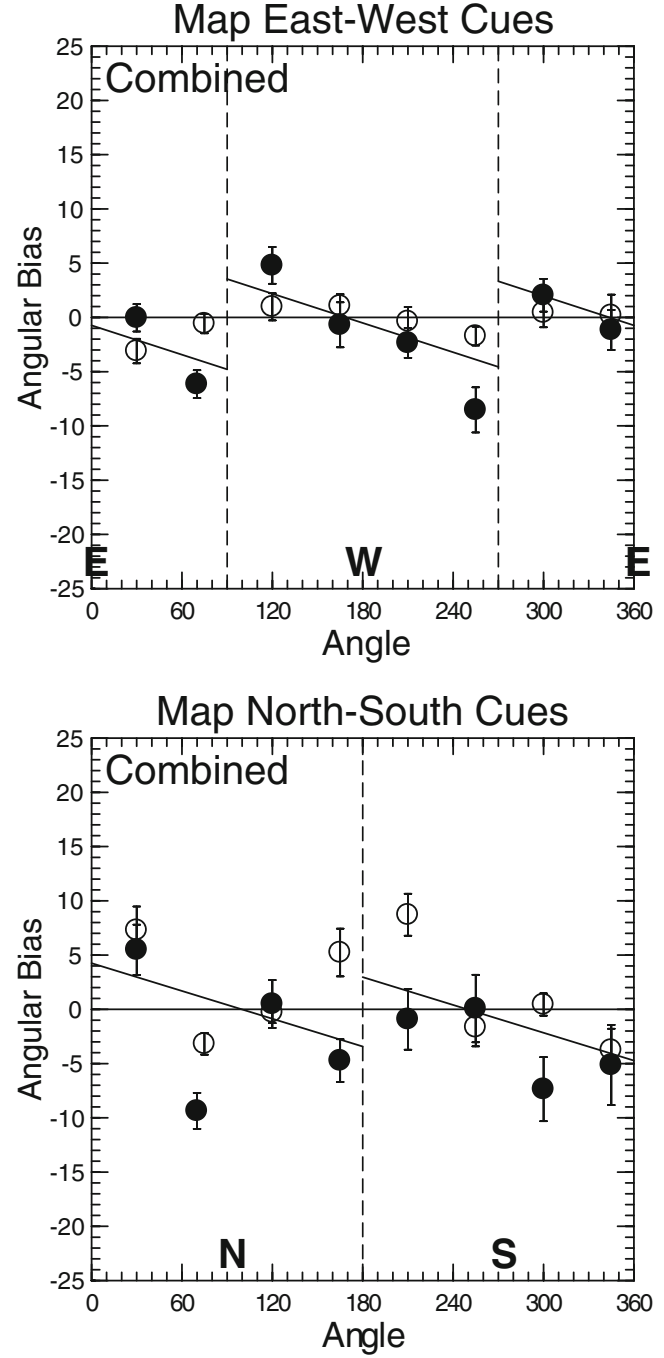

Fig. 5 Mean angular bias (with standard error bars) in the map localization task as a function of target angle and cue location in Experiment 2. Letters (E, W, N, S) reflect locations of east, west, north, and south cues, respectively. Results are generally consistent with two prototypes centered near the cues. Prediction lines are from the fit of the category adjustment model

pattern of angular bias is particularly notable in that it is just the opposite pattern to that obtained in the VE localization task when VE was estimated first. Once again, there is compelling evidence for cue-based spatial partitioning, but in the map localization task, the cues served as prototypes rather than boundaries. This finding is consistent with past results in which requiring participants to transform the representation on some trials led to cue-centered prototypes when locating a target in a small, two-dimensional allocentric task space (Fitting, Wedell \& Allen, 2007a, b, 2008b, 2009).

A 2 (order) $\times 2$ (cues) $\times 2$ (radius) $\times 8$ (angle) repeated measures ANOVA was conducted on angular bias scores for the map localization task. The main effect of angle was significant, $F(7,462)=6.50, p<.001$. There was also a cues $\times$ angle interaction, $F(7,462)=6.23, p<.001, \eta^{2}=.09$, indicating that cues affected the nature of the angular bias. Although the cues $\times$ angle interaction would appear to change across order conditions as shown in Fig. 5, the order $\times$ cues $\times$ angle interaction was not significant, $F(7,462)=$ $1.13, p>.05$. Four other effects reached statistical significance. The main effect of radius, $F(1,66)=20.40, p<.001$, reflected the same effect as that reported for VE estimation, with overall bias scores more positive for the long-radius than for the short-radius targets. The effect of radius on angular bias was moderated by other factors, as described in the significant radius $\times$ angle interaction, $F(7,462)=$ $3.17, p<.01$, and radius $\times$ angle $\times$ cues interaction, $F(7$, $462)=2.75, p<.01$. With regard to the radius $\times$ angle interaction, the positive bias for long-radius targets relative to short-radius targets was greatest for angles $75^{\circ}, 165^{\circ}$, and $210^{\circ}$, moderate for angles $255^{\circ}$ and $300^{\circ}$, and small or slightly reversed for the other target angles. With regard to the radius $\times$ angle $\times$ cues interaction, the effects of radius as shown in Fig. 5 appear greater for the NS cues condition than for the EW cues condition, especially in the bottom portion of the map (angles $180^{\circ}$ to $360^{\circ}$ ). The only other significant effect was a cues $\times$ order interaction, which would appear to reflect the higher overall angular bias scores in the VE-first-NS-cues condition. While the latter four effects appear reliable, they are not particularly relevant to the key hypotheses being tested concerning the cues $\times$ angle interaction.

To better describe the cues $\times$ angle interaction, we fit the basic category adjustment model of Eq. 1 to the angular bias data for each cue condition, combining across order in the same three ways as described earlier, with the regression functions for the model that fit best shown in Fig. 5. For the EW condition, the two-category model with boundaries at $90^{\circ}$ and $270^{\circ}$ fit best, $R^{2}=.555, \lambda=0.955, \rho_{1}=168.60$, and $\rho_{2}=343.69$. For the NS condition, the two-category model with boundaries at $0^{\circ}$ and $180^{\circ}$ fit best, $R^{2}=.203, \lambda=0.957$, $\rho_{1}=99.64$, and $\rho_{2}=249.44$.

Radial bias for VE localization A repeated measures ANOVA of the same form as that described above was conducted on radial bias for VE localization. The only effect to achieve statistical significance was the main effect of radius, $F(1,66)=37.35, p<.001$. While both short- and long-radius targets were underestimated, underestimation was significantly greater for the long-radius targets $(M=-200.14)$ than for the short-radius targets $(M=-129.61)$. These results are consistent with the results of Experiment 1.

Radial bias for map localization A repeated measures ANOVA of the same form as that described above was conducted on radial bias for map localization. Like the analysis of VE localization, the main effect of radius was 
large and significant, $F(1,66)=37.71, \mathrm{p}<.001$. However, unlike for VE localization, both targets were not underestimated, but rather the long-radius targets were underestimated $(M=-2.36)$ and the short-radius targets were overestimated $(M=3.54)$, implying that the radial prototype lies between the long and short target radii. Furthermore, unlike the VE localization data, two additional effects were found to be statistically significant. The main effect of angle, $F(7,462)=5.97, p<.001$, indicates that radial bias was not uniform across target angles. More important, the cues $\times$ angle interaction, $F(7,462)=3.78, p<.001$, indicates that radial bias was affected by changes in the cue location. This interaction reflected a tendency to show a greater positive radial bias for targets closer to a cue. This might result from an anchoring and adjustment strategy in which one remembers the target being near the cue and, hence, underestimates its distance from the cue.

Absolute error for VE localization A repeated measures ANOVA of the same form as that described above was conducted on absolute error for VE localization. As in Experiment 1, there was a large main effect of radius, $F(1,66)=40.07, p<.001$. The average absolute error was greater for long-radius targets $(M=307.16)$ than for short-radius targets $(M=251.26)$. This effect is likely due to the greater radial bias for long-radius targets. The only other statistically significant effect was the main effect of cues, $F(1,66)=7.21, p<.01$, with error greater for the NS condition $(M=298.32)$ than for the EW condition $(M=262.10)$.

Absolute error for map localization A repeated measures ANOVA of the same form as that described above was conducted on absolute error for map localization. Unlike the VE data, the main effect of radius was only marginally significant, $F(1,66)=6.52, p<.02$. Because the radial error in the map condition was more symmetric, the large advantage for the short-radius targets disappeared. Indeed, the mean absolute error was smaller for the long-radius targets $(M=14.46)$ than for the short-radius targets $(M=15.85)$. As in the VE data, there was a significant effect of cues, $F(1$, $66)=15.26, p<.001$, with absolute error greater for the NS condition $(M=17.75)$ than for the EW condition $(M=$ 12.93). The main effect of angle was significant, $F(7,462)$ $=3.62, p<.001$, as was the radius $\times$ angle interaction, $F(7$, $462)=4.87, p<.001$. Additionally, there were two marginally significant interactions involving cues: the angle $\times$ cues interaction, $F(7,462)=2.59, p<.02$, and the radius $\times$ angle $\times$ cues interaction, $F(7,462)=2.42, p<.02$. Both of these interactions were consistent with previous research in which absolute error was reduced for targets closest to the cues (Fitting et al., 2008a, 2008b), with these effects stronger for long-radius than for short-radius targets.

\section{Discussion}

The goals of Experiment 2 were twofold. First, we wished to determine whether the cue-based categorization effects found in the VE of Experiment 1 would replicate when participants were now additionally locating the targets on maps. Second, we wished to examine how labile spatial categorization schemes are across task environments: Would having the same participants localize the same targets within a few seconds of the other task change how cues were used to form categories in map and VE environments? In answer to our first question, the results of Experiment 1 replicated well when the VE localization task occurred first. In both Experiments 1 and 2, this condition led to cue-based categories in which the cues defined the boundaries of spatial categories, with the corresponding prototypes lying in between cue locations. This result held up well in the EW cue condition when VE localization followed map localization. The pattern was altered somewhat in the NS cue condition, in which participants tended to form four quadrant-based prototypes when VE localization followed map localization. This may be because cues in this condition were aligned with the frame in which the map was presented and did not require the participant to use much effort in the translation of space.

In answer to the second question, we observed very different results for map localization and VE localization. These occurred despite the two tasks taking place within seconds of one another, with the same participants locating the same targets, but in a different task environment. Thus, it would appear that spatial categorization is highly dependent on the retrieval context, rather than just the encoding context. The map localization task led to cue-based categorization of a form different from that found with VE localization. Whereas VE localization led to cues being used to define category boundaries, map localization led to cues being used to define category prototypes. The fact that the map-based pattern of bias differed from the corresponding pattern in the VE condition for the same participants reflects the great flexibility in cue-based category use across the tasks.

In addition to these key findings, there were a number of other significant effects found in Experiment 2. Clearly, the pattern of radial bias was very different in map localization and VE localization. In map localization, no transformation of the location was needed and the radial prototype was located between the short- and long-radius targets. In VE localization, there was again strong underestimation of locations. Because participants navigated from the center of the arena, this resulted in severe negative radial bias for both targets. These results are consistent with the underestimation of distance noted in VE distance estimation previously, 
with underestimation increasing with increased distance (Foreman et al., 2004; Hutcheson \& Wedell, 2009).

\section{General discussion}

The overall goal of these two experiments was to investigate the role of cues in estimating locations within a VE from map-based encoding of locations in memory. Our results have important implications for understanding the formation of cue-based categories under the category adjustment model. Taken together, these experiments imply that spatial categorization is a highly flexible process that is strongly influenced by the retrieval environment. When the initial map encoding viewpoint had to be transformed to navigate to the location in the VE at retrieval in Experiments 1 and 2, cues served to define borders of the cue-based spatial categories. However, Experiment 2 results clearly demonstrated a different pattern of cue-based categorization when the retrieval task switched to recall directly on the map, either immediately before or immediately after retrieval via navigation. Prior research indicates that cues should have been ignored and a four-quadrant bias pattern found in the map localization task if the VE task had not been additionally performed (Fitting et al., 2008a). The two estimates from memory were generated within a few seconds of one another, presumably facilitating the use of the same organizational structure in both cases. However, even under these conditions, we found that the pattern of angular bias from the two tasks implied two different spatial category representations.

It is important to note that cues may serve very different purposes when viewed from outside the configuration, rather than from within the configuration. The cues on the map can be considered proximal cues and integrated readily with the location of the target. When inside the large arena, these cues may primarily serve to orient and partition the space. Thus, the cues serve different purposes at retrieval for the two tasks and can be used differently even when the two tasks are conducted right after each other. Recent research by Sargent, Dopkins, and Philbeck (2011) is supportive of the idea that spatial categories can be reorganized at retrieval. The pattern of angular bias they found implied that rotating the participant's egocentric orientation to the task field led to the establishment of new spatial categories centered on the new heading. Furthermore, Sampaio and Wang (2009) found strong evidence for the retrieval basis of angular bias effects, using the dot-in-circle task such that reproduced locations showed the usual bias toward the prototype but recognition tests of location did not. Additional evidence of the flexibility of spatial categories is provided by Verbeek and Spetch (2008), who demonstrated how imposing lines that indicated spatial partitions resulted in a reverse pattern of bias than would have been expected if the lines had been used as borders defining categories.
However, one may question whether the differences we observed between tasks are due to category reorganization at retrieval or simply to differences inherent in the tasks themselves. Recalling the location on the map could be achieved by direct comparison with the encoded image, but navigation to the location in the VE required translation across viewpoints. Tasks also differed in scale and orientation. Future research is needed to clarify the role of these factors.

In both of our experiments, we found evidence of radial bias effects. These effects were largely unrelated to cues but, rather, were tied to how distance estimation depends on the encoding and retrieval environments. Following the pattern found in prior research (Foreman et al., 2004; Hutcheson \& Wedell, 2009), the magnitude of underestimation in navigation increased with increased distance. Experiment 2 demonstrated that the gross underestimation of distance in the VE environment did not generalize to the recall on the map, the original encoding environment. In summary, it appears that translating map distances to VE navigated distances results in gross underestimation of distances. Future research in this area should examine to what degree underestimation in the $\mathrm{VE}$ is due to the navigation process or to changes in the underlying representation.

Finally, the time course of the effects we observed could be investigated in future research using process tracing during navigation in the VE. In their work on navigating a small space on the computer screen, Fitting et al. (2009) found bias in heading error, implicating the use of cue-based categories from the outset of the navigation task. This may or may not be true when navigating in a large VE. Another issue requiring further investigation is the issue of scale. Fitting, Allen, and Wedell (2007a, b) found cue-centered categories in a very small (3-m diameter) traversable space. In such a space, the cues may be proximal rather than distal and, thus, used differently. The present research found cuebased boundaries for a much larger VE space. A VE experiment could systematically manipulate the scale and examine its role in determining categorization of the space.

\section{References}

Diwadkar, V. A., \& McNamara, T. P. (1997). Viewpoint dependence in scene recognition. Psychological Science, 8, 302-307.

Engebretson, P. H., \& Huttenlocher, J. (1996). Bias in spatial location due to categorization: Comment on Tversky and Schiano. Journal of Experimental Psychology. General, 125, 96-108.

Fitting, S., Allen, G. L., \& Wedell, D. H. (2007a). Remembering places in space: A human analog study of the Morris water maze. In T. Barkowsky, M. Knauff, G. Ligozat, \& D. R. Montello (Eds.), Spatial cognition V: Reasoning, action, interaction, LNAI 4387 (pp. 59-75). Berlin: Springer.

Fitting, S., Wedell, D. H., \& Allen, G. L. (2007b). Memory for spatial location: Cue effects as a function of field rotation. Memory \& Cognition, 35, 1641-1658. 
Fitting, S., Wedell, D. H., \& Allen, G. L. (2008a). Cue usage in memory for location when orientation is fixed. Memory \& Cognition, 36, 1196-1216.

Fitting, S., Wedell, D. H., \& Allen, G. L. (2008b). External cue effects on memory for spatial location within a rotated task field. Spatial Cognition and Computation, 8, 219-251.

Fitting, S., Wedell, D. H., \& Allen, G. L. (2009). Cue effects on memory for location when navigating spatial displays. Cognitive Science, 33, 1267-1300.

Foreman, N., Sandamas, G., \& Newson, D. (2004). Distance underestimation in virtual space is sensitive to gender but not activitypassivity or mode of interaction. Cyberpsychology \& Behavior, 7, 451-457.

Friedman, A., \& Waller, D. (2008). View combination in scene recognition. Memory \& Cognition, 36, 467-478.

Friedman, A., Waller, D., Thrash, T., Greenauer, N., \& Hodgson, E. (2011). View combination: A generalization mechanism for visual recognition. Cognition, 119, 229-241.

Greenauer, N., \& Waller, D. (2008). Intrinsic array structure is neither necessary nor sufficient for nonegocentric coding of spatial layouts. Psychonomic Bulletin \& Review, 15, 1015-1021.

Greenauer, N., \& Waller, D. (2010). Micro- and macroreference frames: Specifying the relations between spatial categories in memory. Journal of Experimental Psychology: Learning, Memory, and Cognition, 36, 938-957.

Haun, D. B. M., Allen, G. L., \& Wedell, D. H. (2005). Bias in spatial memory: A categorical endorsement. Acta Psychologica, 118, 149-170.

Hund, A. M., \& Plumert, J. M. (2002). Delay-induced bias in children's memory for location. Child Development, 73, 829-840.

Hutcheson, A. T., \& Wedell, D. H. (2009). Moderating the route angularity effect in a virtual environment: Support for a dual memory representation. Memory \& Cognition, 37, 514-521.

Huttenlocher, J., Hedges, L. V., Corrigan, B., \& Crawford, L. E. (2004). Spatial categories and the estimation of location. Cognition, 93, 75-97.

Huttenlocher, J., Hedges, L. V., \& Duncan, S. (1991). Categories and particulars: Prototype effects in estimating spatial location. Psychological Review, 98, 352-376.

Ishikawa, T., \& Montello, D. R. (2006). Spatial knowledge acquisition from direct experience in the environment: Individual differences in the development of metric knowledge and the integration of separately learned places. Cognitive Psychology, 52, 93-129.

Learmonth, A. E., Nadel, L., \& Newcombe, N. S. (2002). Children's use of landmarks: Implications for modularity theory. Psychological Science, 13, 337-341.

Learmonth, A. E., Newcombe, N. S., \& Huttenlocher, J. (2001). Toddler's use of metric information and landmarks to reorient. Journal of Experimental Child Psychology, 80, 225-244.
Learmonth, A. E., Newcombe, N. S., Sheridan, N., \& Jones, M. (2008). Why size counts: Children's spatial reorientation in large and small enclosures. Developmental Science, 11, 414-426.

Mou, W., \& McNamara, T. P. (2002). Intrinsic frames of reference in spatial memory. Journal of Experimental Psychology: Learning, Memory, and Cognition, 28, 162-170.

Mou, W., McNamara, T. P., Rump, B., \& Xiao, C. (2006). Roles of egocentric and allocentric spatial representations in locomotion and reorientation. Journal of Experimental Psychology: Learning, Memory, and Cognition, 32, 1274-1290.

Sampaio, C., \& Wang, R. F. (2009). Category-based errors and the accessibility of unbiased spatial memories: A retrieval model. Journal of Experimental Psychology: Learning, Memory, and Cognition, 5, 1331-1337.

Sargent, J., Dopkins, S., \& Philbeck, J. (2011). Dynamic category structure in spatial memory. Psychonomic Bulletin \& Review, $18,1105-1112$.

Shelton, A. L., \& McNamara, T. P. (2004). Orientation and perspective dependence in route and survey learning. Journal of Experimental Psychology: Learning, Memory, and Cognition, 30, 158-170.

Shelton, A. L., \& Pippitt, H. A. (2007). Fixed versus dynamic orientations in environmental learning from ground-level and aerial perspectives. Psychological Research, 71, 333-346.

Siegel, A. W., \& White, S. H. (1975). The development of spatial representations of large-scale environments. Advances in Child Development and Behavior, 10, 10-55.

Spencer, J. P., \& Hund, A. A. (2002). Prototypes and particulars: Geometric and experience-dependent spatial categories. Journal of Experimental Psychology. General, 131, 16-37.

Taylor, H. A., Naylor, S. J., \& Chechile, N. A. (1999). Goal-specific influences on the representation of spatial perspective. Memory \& Cognition, 27, 309-319.

Thorndyke, P. W., \& Hayes-Roth, B. (1982). Differences in spatial knowledge acquired from maps and navigation. Cognitive Psychology, 14, 560-589.

Verbeek, E., \& Spetch, M. (2008). Distortions in location memory. Psychonomic Bulletin \& Review, 15, 328-336.

Waller, D. (2006). Egocentric and nonegocentric coding in memory for spatial layout: Evidence from scene recognition. Memory \& Cognition, 34, 491-504.

Waller, D., Friedman, A., Hodgson, E., \& Greenauer, N. (2009). Learning scenes from multiple views: Novel views can be recognized more efficiently than learned views. Memory \& Cognition, 37, 90-99.

Wedell, D. H., Fitting, S., \& Allen, G. L. (2007). Shape effects on memory for location. Psychonomic Bulletin \& Review, 14, 681-686.

Witmer, B. G., \& Sadowski, W. J. (1998). Nonvisually guided locomotion to a previously viewed target in real and virtual environments. Human Factors, 40, 478-488. 